\title{
Integral Relations Among Bessel Functions
}

\section{By E. O. Schulz-DuBois}

\begin{abstract}
Inverse Laplace transformation of seemingly trivial identities is used to derive
\end{abstract} novel integral relations among ordinary and modified Bessel functions.

Consider the seemingly trivial identity

$$
\exp (+a / p) \exp (-a / p)=1, a>0 \text {, real ; }
$$

where $p$ denotes the Laplace transform variable. This relation is of some significance in the theory of wave propagation in linear dispersive media, where it relates to the restoration of distorted signals [1]. For convenience Eq. (1) may be given in the more extended form

$$
\begin{aligned}
{[\exp (+a / p)-1][\exp (-a / p)-1]=} & {[1-\exp (+a / p)] } \\
+[1-\exp (-a / p)] &
\end{aligned}
$$

If one applies standard rules of inverse Laplace transformation [2] to Eq. (1a), one obtains

$$
\begin{aligned}
\left(\frac{a}{t}\right)^{1 / 2} & \left(I_{1}\left((4 a t)^{1 / 2}\right)-J_{1}\left((4 a t)^{1 / 2}\right)\right) \\
= & \int_{0}^{t}\left(\frac{a}{t-\tau}\right)^{1 / 2}\left(\frac{a}{\tau}\right)^{1 / 2} I_{1}\left((4 a(t-\tau))^{1 / 2}\right) J_{1}\left((4 a \tau)^{1 / 2}\right) d \tau,
\end{aligned}
$$

which by variable substitution assumes the more convenient forms

$$
\begin{aligned}
\frac{I_{1}(x)-J_{1}(x)}{x} & =\int_{0}^{x} \frac{I_{1}\left(\left(x^{2}-\xi^{2}\right)^{1 / 2}\right) J_{1}(\xi)}{\left(x^{2}-\xi^{2}\right)^{1 / 2}} d \xi \\
& =\int_{0}^{\pi / 2} J_{1}(x \sin \vartheta) I_{1}(x \cos \vartheta) d \vartheta
\end{aligned}
$$

This relation appears to be novel. Its derivation from similar integral relations in the literature requires a nontrivial effort. During examination of the manuscript, two referees derived Eq. (3b) independently. Their approach will now be sketched.

The first is as follows: Expand $I_{1}$ on the right-hand side of Eq. (3b) in powers of $\cos \theta$. Integrate term by term using Eq. (2) on p. 374 of Watson's book [3]. Apply the definition of the Lommel functions $s_{\mu, \nu}(z)$ from p. 345 of Watson and carry out the summation, allowing for the identity Eq. (9) on p. 141 of Watson. This yields the left-hand side of the present Eq. (3).

The second approach starts from Eq. (25) on p. 299 of the book by Luke [4]. The integral of this formula reduces to the present integral by the choice $\mu=\nu=$ $\alpha=\beta=\frac{1}{2}, w=i z$. Luke's formula gives the result as an infinite sum over generalized hypergeometric functions. In the present case the series expansion of these functions

Received December 17, 1968, revised March 31, 1969. 
terminates, since at least one of the upper indices is a negative integer. The original generalized hypergeometric functions ${ }_{3} F_{2}$ may be reduced to functions of the type ${ }_{2} F_{1}$ and then to elementary expressions involving only integers. The resulting series can be shown to represent the left-hand side of the present Eq. (3).

It should be noted that Eq. (3) is not a special case of the formula Eq. (31) on p. 301 of Luke.

Other integral identities may be generated by inverse Laplace transformation of similarly trivial identities. For example, from

$$
\exp (a / p) \exp (a / p)=\exp (2 a / p)
$$

and its reciprocal

$$
\exp (-a / p) \exp (-a / p)=\exp (-2 a / p)
$$

one finds

$$
2^{1 / 2} I_{1}\left(2^{1 / 2} x\right)-2 I_{1}(x)=x \int_{0}^{\pi / 2} I_{1}(x \sin \vartheta) I_{1}(x \cos \vartheta) d \vartheta
$$

and

$$
2 J_{1}(x)-2^{1 / 2} J_{1}\left(2^{1 / 2} x\right)=x \int_{0}^{\pi / 2} J_{1}(x \sin \vartheta) J_{1}(x \cos \vartheta) d \vartheta
$$

Similar integral relations are obtained by considering transformations leading to other orders of Bessel functions [5]. For example, from

$$
\begin{aligned}
(1 / p) \exp (-a / p) \exp (a / p) & =1 / p \\
(1 / p) \exp (a / p) \exp (-a / p) & =1 / p \\
(1 / p) \exp (a / p) \exp (a / p) & =(1 / p) \exp (2 a / p) \\
(1 / p) \exp (-a / p) \exp (-a / p) & =(1 / p) \exp (-2 a / p)
\end{aligned}
$$

one obtains after transformation

$$
\begin{aligned}
& \int_{0}^{\pi / 2} J_{0}(x \cos \vartheta) I_{1}(x \sin \vartheta) x \cos \vartheta d \vartheta=1-J_{0}(x) \\
& \int_{0}^{\pi / 2} I_{0}(x \cos \vartheta) J_{1}(x \sin \vartheta) x \cos \vartheta d \vartheta=I_{0}(x)-1 \\
& \int_{0}^{\pi / 2} I_{0}(x \cos \vartheta) I_{1}(x \sin \vartheta) x \cos \vartheta d \vartheta=I_{0}\left(2^{1 / 2} x\right)-I_{0}(x) \\
& \int_{0}^{\pi / 2} J_{0}(x \cos \vartheta) J_{1}(x \sin \vartheta) x \cos \vartheta d \vartheta=J_{0}(x)-J_{0}\left(2^{1 / 2} x\right) .
\end{aligned}
$$

In general, these and similar integral relations among Bessel functions may be generated by inverse Laplace transformation of functions of the type

$$
f(p) \cdot g(p)=f_{1}(p)+g_{1}(p) .
$$

In the example of Eq. (1a) above, $f=f_{1}$ and $g=g_{1}$, but this need not be the case. 
It is perhaps surprising that the powerful methods of Laplace transformation were not employed more frequently in the development of the theory of Bessel functions. This probably has historical reasons in that the theory of Bessel functions was virtually completed before the theory of Laplace transforms was developed.

The author acknowledges stimulating discussions with Dr. R. A. Toupin of the IBM Thomas J. Watson Research Center. He also acknowledges lucid comments from two referees.

IBM Zurich Research Laboratory

8803 Rüschlikon, Switzerland

1. E. O. Schulz-DuBors, Electromagnetic Propagation in Linear Dispersive Media. (To appear.)

2. A. H. Zemanian, Distribution Theory and Transform Analysis. An Introduction to Generalized Functions, with Applications, McGraw-Hill, New York, 1965, Table B.2, formulae 64, 84, 96. MR $31 \# 1556$.

3. G. N. Watson, A Treatise on the Theory of Bessel Functions, 2nd ed., Cambridge Univ. Press, New York, and Macmillan, New York, 1944. MR 6, 64.

4. Y. L. Luke, Integrals of Bessel Functions, McGraw-Hill, New York, 1962. MR 25 \#5198.

5. A. ERdélyi et al., Higher Transcendental Functions, Vol. 2, McGraw-Hill, New York, 1953, pp. 244-245, formulae 31,35. MR 15, 419. 